\section{Die Entwicklung der psychischen Gesundheit in der heroingestützten Behandlung - Ergebnisse aus der schweizerischen Studie PROVE}

Zusammenfassung: Hintergrund: In den Versuchen für eine ärztliche Verschreibung von Betäubungsmitteln (PROVE) wurden in den Jahren 1994-96 für die heroingestützte Behandlung 800 Plätze zur Verfügung gestellt. Im Rahmen der wissenschaftlichen Begleitevaluation wurde untersucht, inwieweit dieses Behandlungsprogramm Schwerstabhängige einer Behandlung zuführen kann, welche durch die bisherigen Therapiemöglichkeiten nicht ausreichend erreicht worden sind. In dieser Arbeit wird die psychische Situation der Patienten in der heroingestützten Behandlung beschrieben.

Methode: Aufnahmebedingungen waren ein Mindestalter von 20 Jahren, mindestens seit zwei Jahren täglicher Opiatkonsum, mindestens zwei vorgängige, erfolglose Behandlungen und nachgewiesene soziale und/oder medizinische Defizite in Folge der Opiatabhängigkeit. Bei Eintritt und alle 6 Monate wurde die psychische Gesundheit von den behandelnden Ärzten untersucht. Externe Interviewerinnen befragten die Patienten zu ihren psychischen Symptomen.

Ergebnisse: Bei Eintritt waren $41 \%$ der Patienten in einem schlechten psychischen Zustand. Es zeigte sich eine hohe Komorbiditätsrate. Während der Behandlung wurden bei $9 \%$ der Patienten eine Schizophrenie, bei 39\% affektive Störungen und bei $68 \%$ eine Persönlichkeitsstörung diagnostiziert. Der allgemeine psychische Zustand verbesserte sich während der Behandlung. So halbierte sich die Anzahl Patienten in schlechtem psychischen Zustand schon nach wenigen Monaten. Entsprechend gaben die Patienten auch weniger psychische Beschwerden an und die Zahl der Spitaleinweisungen ging um $59 \%$ zurück.

Schlussfolgerung: Bekanntermaßen treten bei intravenös Drogenabhängigen vermehrt psychische Störungen auf. Die schweizerischen Versuche für eine ärztliche Verschreibung von Betäubungsmitteln erreichten eine große Gruppe von Patienten mit solchen psychischen Störungen und Symptomen.Während der heroingestützten Behandlung zeigte sich eine erhebliche Verbesserung der psychischen Gesundheit dieser Patienten. Zukünftig sollte aufbauend auf den bisherigen Erfahrungen die psychiatrische Diagnostik und Therapie für diese ambulanten Patienten weiter optimiert werden.

Schlüsselwörter: Drogenabhängigkeit - Heroin - heroingestützte Behandlung - psychische Gesundheit - komorbide psychische Störung

Suchttherapie 2000; $1: 27-33$

(c) Georg Thieme Verlag Stuttgart · New York

ISSN 1439-9903
Thomas Steffen ${ }^{1,2}$, Toni Berthel ${ }^{3}$, Alexander Zimmer ${ }^{4}$, Felix Gutzwiller ${ }^{2}$, Ambros Uchtenhagen ${ }^{1}$

1 Institut für Suchtforschung, Zürich

${ }^{2}$ Institut für Sozial- und Präventivmedizin der Universität Zürich

${ }^{3}$ Behandlungsstelle HeGeBe, Winterthur

${ }^{4}$ Behandlungsstelle HeGeBe, Olten und Solothurn

Development of Mental Health in Heroin-Assisted Treatment: Results from the Swiss PROVE Study: Objectives: During 1994-96 some 800 slots were made available for heroin-assisted treatment within trials of medical prescription of narcotics (PROVE). Studies carried out with scientifically monitored assessment examined the extent to which the most severely addicted can be guided to therapeutic options when all previous efforts to reach them have proved insufficient. This study describes the mental health of patients in heroin-assisted treatment.

Methods: Entry criteria were minimal age of 20, minimal duration of daily opiate consumption of 2 years, negative outcome of at least 2 previous treatments, documented social and/or health deficits as a consequence of their opiate dependence. On admission and during all six month of treatment, the state of mental health was examined by physicians. External interviewers asked the patients about their mental symptoms.

Results: On admission, $41 \%$ of patients were found in a poor mental state. The mental-addictive disorder comorbidity rate was high. The treatment prevalence of schizophrenia was $9 \%$, of affective disorders $39 \%$, of personality disorders $68 \%$. The general state of mental health improved during treatment. The number of patients in mental state was already reduced by half after a few months. Accordingly the patients also indicated less mental disorders, and the number of hospital referrals declined by $59 \%$.

Conclusions: As is well known, mental disorders arise at an increased rate among intravenous drug addicts. The Swiss trials of medical prescription of narcotics reached a large group of patients with such disorders and symptoms. During the heroin-assisted treatment, a substantial improvement occurred in the mental health of these patients. In future, based on previous experience, psychiatric diagnosis and therapy for these out-patients should be improved even more.

Key words: Drug Addiction - Heroin - Heroin-Assisted Treatment - Mental Health - Comorbidity of Psychiatric Disorders

\section{Einleitung}

Hintergrund

Für das einzelne Individuum wie auch für die Gesellschaft sind mit der Drogenabhängigkeit häufig ganz erhebliche gesundheitliche und soziale Probleme verbunden. Typisch ist eine ungünstige soziale Situation, welche sich beispielsweise 
in einem ungenügenden sozialen Netz, einer hohen Delinquenzrate und ungünstigen Wohn- und Arbeitsbedingungen zeigt. Die Prävalenz verschiedener psychischer und somatischer Erkrankungen ist bei Drogenkonsumierenden deutlich erhöht. Das gemeinsame Auftreten von sozialen, somatischen und psychischen Problemen stellt sehr hohe Anforderungen an die medizinische Behandlung [1]. Häufig fehlen die geeigneten, systematisch erhobenen Basisinformationen, um die individuelle Diagnostik und Therapie, aber auch die Behandlungsangebote auf der Systemebene weiterzuentwickeln.

Bei komplexen Krankheitsbildern wie den psychischen Störungen bei Opiatabhängigen sind die geforderten, aktuellen Daten, die zur gezielten Behandlungsoptimierung und zur Systementwicklung nötig sind, häufig nicht oder nur unvollständig vorhanden.

Die hier vorliegende Arbeit möchte vor diesem Hintergrund die psychische Gesundheit ambulanter Patienten in der heroingestützten Behandlung in der Schweiz beschreiben. Neben der Darstellung der Eintrittssituation wird auf die Frage der Komorbidität und auf die Entwicklung innerhalb der heroingestützten Behandlung besonders eingegangen.

\section{Psychische Störung und Opiatabhängigkeit}

Bei der Diskussion der Faktoren, welche eine Abhängigkeitsentwicklung begünstigen, ist in den letzten Jahren verstärkt die Bedeutung von psychischen Störungen und Symptomen erörtert worden. So zeigen verschiedene internationale Studien, dass rund jeder zweite Drogenabhängige während seines Lebens von psychischen Störungen nach ICD-10 oder DSM-IV betroffen ist. Diese Rate ist zwei- bis dreimal so hoch wie in der Allgemeinbevölkerung [2].

Eine aus epidemiologischer Sicht besonders bedeutende Studie hierzu ist die „Epidemiological Catchment Area (ECA) Study“, welche anfangs der 80er Jahre in den USA durchgeführt wurde. Untersucht wurden über 20000 Personen ab 18 Jahren [3,4]. In dieser großen epidemiologischen Studie wurde eine Lifetime-Prävalenz von $23 \%$ für eine psychiatrische Erkrankung, von 14\% für eine Alkoholabhängigkeit bzw. einen Alkoholmissbrauch und von 6\% für eine andere Drogenabhängigkeit bzw. einen Drogenmissbrauch in der Allgemeinbevölkerung gefunden. Das Risiko einer Alkoholabhängigkeit war bei Patienten mit einer Schizophrenie um das Dreifache erhöht, das Risiko einer anderen Substanzabhängigkeit war bei diesen Patienten rund sechsmal höher. In der ECA-Studie zeigte sich auch ein gehäuftes Auftreten von unipolaren Depressionen bei nicht alkoholischen Substanzabhängigkeiten. Die affektiven Störungen insgesamt lagen bei einer Prävalenz von $8 \%$, wovon $32 \%$ eine zusätzliche Substanzabhängigkeit bzw. einen Substanzmissbrauch aufwiesen. Weiter wurde bei $61 \%$ der Patienten mit bipolaren Störungen ein Substanzmissbrauch festgestellt, wobei Alkohol mit $46 \%$ die am meisten gebrauchte Substanz war. In der ECA-Studie fand man ferner bei $84 \%$ der Personen mit einer antisozialen Persönlichkeitsstörung eine Form von Substanzmissbrauch. Bei Patienten mit antisozialen Persönlichkeitsstörungen war das Risiko für Alkoholismus um den Faktor 21 und für Drogenmissbrauch um den Faktor 13 erhöht.
Zusammenfassend kann festgestellt werden, dass die psychische Komorbidität gemäß den heute vorliegenden Studien bei Opiatabhängigen weit verbreitet ist [5]. Für die Public-HealthPlanung der Behandlung und Intervention im Drogenbereich stellt sich damit eine besondere Herausforderung. Die Behandlung dieser Drogenabhängigen mit zusätzlichen psychischen Problemen muss aufgrund der komplexen therapeutischen Situation fachübergreifend in einem Behandlungssetting stattfinden. Häufig fehlt aber für ein solches multiprofessionelles Vorgehen, wie erwähnt, noch die nötige Wissens- und Versorgungsstrukturbasis [2].

\section{Die heroingestützte Behandlung in der Schweiz}

In der Schweiz wurden seit Mitte der achtziger Jahre verschiedene Maßnahmen zur Verbesserung der Situation im Drogenbereich ergriffen. So wurden für die Behandlung von Drogenabhängigen in der Schweiz verschiedenste Therapieformen ausgebaut. Rund 1700 Behandlungsplätze in stationären Einrichtungen stehen heute zur Suchtentwöhnung und -rehabilitation zur Verfügung. Über 15000 Opiatabhängige erhalten gegenwärtig im Rahmen von Substitutionsbehandlungen Methadon. Mit den bestehenden Hilfs- und Behandlungsprogrammen konnte aber nur ein Teil der rund 30000 Opiatabhängigen erreicht werden. Vor allem Abhängige mit besonders schweren sozialen und medizinischen Defiziten blieben häufig ohne Behandlung. Vor diesem Hintergrund wurden die Versuche für eine ärztliche Verschreibung von Betäubungsmitteln (PROVE), in welchen die heroingestützte Behandlung untersucht wurde, begonnen.

Im Vorfeld dieser Studien zur heroingestützten Behandlung wurden die bisherigen veröffentlichten Studien zur Opiatsubstitution systematisch recherchiert [6]. Die durchgeführte Literaturanalyse zeigte erhebliche Wissenslücken bezüglich der möglichen Anwendung der verschiedenen Opiate innerhalb von medizinischen Behandlungen.

Ab 1994 konnte im Rahmen der schweizerischen Versuche für eine ärztliche Verschreibung von Betäubungsmitteln (PROVE) die heroingestützte Behandlung in einer Kohortenstudie, welche in den ersten 30 Monaten über 1035 Patienten einschloss, untersucht werden $[7,8,9]$. Eine Vielzahl von neuen Erkenntnissen über die Verschreibung von Heroin an Opiatabhängige konnte auf diese Weise systematisch erarbeitet werden. Diese Forschungsresultate haben zu einer breiteren internationalen Diskussion der heroingestützten Behandlung geführt $[10,11,12,13,14]$.

In diesem Artikel werden Resultate zur psychischen Gesundheit der teilnehmenden Patienten der ersten vier Studienjahre dargestellt und die laufenden Bestrebungen zur Behandlungsoptimierung diskutiert.

\section{Methodik}

\section{Studienteilnehmer}

Von anfangs 1994 bis zum 30. Juni 1996 wurden insgesamt 1146 Patienten in die heroingestützte Behandlung aufgenommen. Davon konnten 1035 Patienten in der wissenschaftlichen Kohortenstudie untersucht werden. 111 Eintretende wurden aus der Analyse aus folgenden Gründen ausgeschlos- 
sen: 84 traten vor Abschluss der Eintrittsuntersuchung aus der Behandlung aus und 27 erfüllten die methodischen Einschlusskriterien nicht.

30\% der in die Behandlung Eintretenden waren Frauen und 70\% Männer. Das Durchschnittsalter betrug bei Eintritt 31 Jahre. Aufgenommen wurden i.v. Drogenabhängige in 17 mehrheitlich städtischen Projektstellen in der Schweiz.

Die hier beschriebene, für die Behandlungskohorte repräsentative Verlaufsstichprobe umfasst alle 237 Patienten, welche zwischen dem 1.1.94 und dem 31.3.95 ins Programm eingetreten sind und während mindestens 18 Monaten in der heroingestützten Behandlung verblieben. 128 Patienten, die im Zeitraum vom Januar 1994 bis März 1995 eingetreten sind, verließen die Studie vor dem 18 Behandlungsmonat. Davon sind $57 \%$ in eine Anschlussbehandlung (Methadonsubstitution, abstinenzorientierte Behandlung, Entzug) übergetreten.

Für die Analyse der Komorbidität wurden die Patienten aus zwei Behandlungsstellen (Solothurn und Winterthur) im Jahre 1997/8 zusätzlich untersucht. Erhoben wurde die aktuelle Prävalenz von psychischen Störungen nach ICD-10 bei allen 77 Patienten dieser zwei Behandlungsstellen. Die Diagnose wurde von erfahrenen Psychiatern aufgrund einer aktuellen psychiatrischen Untersuchung unter Einbeziehung von Fremdanamnesen, Umfeldbeobachtungen und der Krankengeschichte gestellt.

Die Daten zur Analyse der Hospitalisationen vor und während der heroingestützten Behandlung wurden 1997/98 erfasst. Untersucht wurden alle 651 Patienten, die zu diesem Zeitpunkt mindestens zwei Jahre in der heroingestützten Behandlung waren. Erfasst wurden die Spitaleinweisungen über eine Patientenbefragung unter Einbeziehung der Krankenakten. Erhoben wurden alle Hospitalisationen, die in den letzten zwei Jahren vor und den ersten zwei Jahren während der heroingestützten Behandlung erforderlich wurden. Stichprobenweise wurden die Ergebnisse mittels der Abrechnungsdaten der obligatorischen Krankenkasse überprüft.

\section{Einschlusskriterien}

Die Aufnahmekriterien waren: ein Mindestalter von 20 Jahren, die Opiatabhängigkeit musste mindestens zwei Jahre betragen und mindestens zwei vorgängige Behandlungsversuche mussten gescheitert sein. Die Abhängigkeit musste zu erheblichen medizinischen und/oder sozialen Defiziten geführt haben.

Die Eintrittskriterien wurden für jeden Teilnehmenden vor Behandlungsbeginn festgehalten. Es wurden nur Personen aufgenommen, welche ihr schriftliches Einverständnis zur Behandlung und wissenschaftlichen Untersuchung gaben.

Die Untersuchung der Teilnehmenden erfolgte nach einem exakten Studienprotokoll. Erhoben wurden soziale, medizinische und pharmakologische Daten der Patienten.

\section{Soziale Daten}

Bei den sozialen Befragungen wurden Teile von eingeführten Fragebogen (EuropASI) verwendet [15]. Die Befragung er- folgte durch von den Behandlungsstellen unabhängige Interviewerinnen in standardisierter Form. Eine regelmäßige Schulung und Validierung der Interviewerinnen wurde durchgeführt.

\section{Medizinisch-pharmakologische Daten}

Die Patienten wurden bei Eintritt und jeden 6. Behandlungsmonat durch die behandelnden Ärzte umfassend medizinisch untersucht. Erhoben wurden in standardisierter Form der somatische und psychische Status der Patienten. Akute medizinische Ereignisse wurden laufend erfasst. Die medizinischen Diagnosen wurden gemäß ICD-10-Regelwerk kodiert [16]. Bei der Erfassung der Selbstangaben zur psychischen Gesundheit wurden 62 Items der SCL-90-Symptomcheckliste verwendet [17].

\section{Behandlung}

Die Behandlung wurde gemäß individueller Indikation festgelegt. Verordnet wurde vorwiegend Heroin neben Methadon und Morphin (tägliche Durchschnittsdosis Heroin i.v. $471 \mathrm{mg}$, Methadon i.v. $87 \mathrm{mg}$, Morphin i.v. $442 \mathrm{mg}$ ). Die Applikation erfolgte intravenös und oral. In der ersten Studienphase wurde einer kleineren Gruppe von Patienten auch Heroin in rauchbarer Form verordnet [8,9]. Die Patienten erhielten in den Projektstellen eine intensive sozialmedizinische Betreuung. Die Studienteilnehmer wurden regelmäßig medizinisch untersucht und somatisch und psychisch behandelt. Sozialhilfe wurde angeboten. Auf hundert behandelte Patienten kamen durchschnittlich 6,6 Vollzeitstellen (Sozialarbeit 2,0 Stellen, ärztlicher Dienst 1,8 Stellen, Administration 1,65 Stellen, Abgabeteam 0,75 Stellen, weitere Dienste 0,4 Stellen) [18]. Die Bedeutung dieser psycho-sozialen und medizinischen Begleitbehandlung wird gegenwärtig vertieft untersucht [19].

\section{Statistik}

Phi-, Cramers-V- und Sign-Test wurden verwendet zur Analyse von Unterschieden zwischen Gruppen. Die Faktoranalyse wurde zur Untersuchung der Variablen der subjektiven Einschätzung der psychischen Gesundheit gebraucht (SCL-90). Aus 62 Items des SCL-90-Fragebogens wurden mittels Faktoranalyse drei Syndrome extrahiert. Die drei Syndrome betrafen Depression, Angst und wahnhafte Beschwerden sowie aggressives Agieren. Die Kohorte wurde anhand der gefundenen Werte bei Eintritt in drei gleich große Gruppen mit den jeweiligen Ausprägungsgraden „tief“, „mittel“ und „hoch“ pro Syndrom eingeteilt. Der Verlauf, bezogen auf die Eintrittseinteilung, wurde analysiert. Als signifikant wird eine Irrtumswahrscheinlichkeit von 5\% und kleiner bezeichnet. Zur statistischen Berechnung wurde das Statistikprogramm SPSS verwendet [20].

\section{Resultate}

\section{Sozialmedizinische Eintrittscharakteristika}

Tab. 1 gibt die sozio-demographischen Charakteristika der Patienten bei Eintritt in die heroingestützte Behandlung der Jahre 1994 bis 1996 wieder. Die Eintretenden wiesen verschiedenste soziale Probleme auf. Rund die Hälfte der Neueintretenden hatte eine instabile Wohnsituation. Nur 16\% gingen 
Tab. 1 Sozio-demographische Eintritts-Charakteristika ( $\mathrm{n=1035)}$

\begin{tabular}{|c|c|}
\hline $\begin{array}{l}\text { Geschlecht } \\
\text { Männer } \\
\text { Frauen }\end{array}$ & $\begin{array}{l}70 \% \\
30 \% \\
\mathrm{md}=0^{a}\end{array}$ \\
\hline $\begin{array}{l}\text { Alter } \\
\text { unter } 25 \text { Jahren alt } \\
26-35 \text { Jahre alt } \\
\text { über } 35 \text { Jahre alt } \\
\text { Durchschnittsalter }\end{array}$ & $\begin{array}{l}19 \% \\
61 \% \\
20 \% \\
30,8 \text { Jahre (SD 5,7) } \\
m d=1\end{array}$ \\
\hline $\begin{array}{l}\text { Wohnsituation } \\
\text { stabil } \\
\text { nicht stabil }\end{array}$ & $\begin{array}{l}51 \% \\
49 \% \\
\mathrm{md}=5\end{array}$ \\
\hline $\begin{array}{l}\text { Freundeskreis } \\
\text { keine Freunde } \\
\text { Drogenabhängige } \\
\text { Freunde ohne Drogen }\end{array}$ & $\begin{array}{l}30 \% \\
30 \% \\
41 \% \\
\mathrm{md}=11\end{array}$ \\
\hline $\begin{array}{l}\text { Arbeit } \\
\text { reguläre Arbeit } \\
\text { Temporärarbeit, Hausarbeit, Rente } \\
\text { arbeitslos }\end{array}$ & $\begin{array}{l}16 \% \\
42 \% \\
42 \% \\
\mathrm{md}=17\end{array}$ \\
\hline $\begin{array}{l}\text { Schulden } \\
\text { keine Schulden } \\
\text { Schulden }\end{array}$ & $\begin{array}{l}17 \% \\
83 \% \\
m d=23\end{array}$ \\
\hline $\begin{array}{l}\text { Finanzielle Unterstützung } \\
\text { Geld vom Sozialamt } \\
\text { Leistungen der Invalidenversicherung }\end{array}$ & $\begin{array}{l}51 \% \mathrm{md}=15 \\
21 \% \mathrm{md}=15\end{array}$ \\
\hline $\begin{array}{l}\text { Szenenkontakt in den letzten } \\
30 \text { Tagen }\end{array}$ & $\begin{array}{l}87 \% \\
\mathrm{md}=15\end{array}$ \\
\hline $\begin{array}{l}\text { Legalverhalten } \\
\text { Verurteilungen seit Beginn mit } \\
\text { Drogenkonsum } \\
\text { illegales Einkommen/Grauzonen- } \\
\text { einkommen }\end{array}$ & $\begin{array}{l}84 \% \mathrm{md}=5 \\
68 \% \mathrm{md}=0\end{array}$ \\
\hline $\begin{array}{l}\text { Suchtmittelkonsum } \\
\text { fast täglicher Heroinkonsum } \\
\text { fast täglicher Kokainkonsum } \\
\text { fast täglicher Benzodiazepinkonsum } \\
\text { regelmäßiger Alkoholkonsum } \\
\text { regelmäßiger Tabakkonsum } \\
\text { Heroinkonsum seit }\end{array}$ & $\begin{array}{l}81 \% \mathrm{md}=3 \\
25 \% \mathrm{md}=3 \\
21 \% \mathrm{md}=3 \\
32 \% \mathrm{md}=32 \\
99 \% \mathrm{md}=3 \\
10,5 \text { Jahren (SD 5,5) } \mathrm{md}=4\end{array}$ \\
\hline $\begin{array}{l}\text { frühere Suchtbehandlungen } \\
\text { Entzugsbehandlungen } \\
\text { Substitutionsbehandlungen } \\
\text { stationäre Therapien }\end{array}$ & $\begin{array}{l}89 \% \mathrm{md}=9 \\
91 \% \mathrm{md}=9 \\
51 \% \mathrm{md}=9\end{array}$ \\
\hline
\end{tabular}

amd= missing data

einer regulären Arbeit nach. Sehr häufig waren Schulden und Illegalverhalten. Neben dem Opiatkonsum wurden verschiedene andere Suchtmittel oft auch regelmäßig konsumiert.

Entsprechend den Eintrittskriterien wiesen die Patienten viele frühere Suchtbehandlungen auf. So hatten 91\% der Eintretenden schon mindestens eine Substitutionsbehandlung durchlaufen. Bei 89\% wurde schon mindestens eine Entzugsbehandlung durchgeführt. Gemäß der serologischen Untersuchung waren 15\% der Eintretenden HIV-positiv, 73\% Hepatitis-B-positiv und $82 \%$ Hepatitis-C-positiv.
Tab. 2 Allgemeine psychische Situation bei Eintritt $(n=1035)$

Psychischer Zustand nach ärztlicher Beurteilung

gut

schlecht

$59 \%$

$41 \%$

$\mathrm{md}=39$

Psychische Probleme in den letzten 30 Tagen

mehr als 3 Tage

mehr als 15 Tage

$47 \%$

$20 \%$

$\mathrm{md}=155$

Arztkontakt in den letzten sechs Monaten wegen psychischer Beschwerden

$22 \%$

$\mathrm{md}=9$

regelmäßige Medikamente wegen psychischer

Erkrankung

$20 \%$

$\mathrm{md}=16$

Rente wegen psychischem Leiden

$13 \%$

$\mathrm{md}=10$

Tab. 3 Anamnestische Angabe zu psychischen Problemen bei Eintritt $(n=1035, \mathrm{md}=16)$

\section{Depression}

schwere Depression insgesamt $\quad 50 \%$

schwere Depression in den letzten 30 Tagen $\quad 19 \%$

Angst- und Spannungszustände

schwere Angst- und Spannungszustände insgesamt $\quad 39 \%$

schwere Angst- und Spannungszustände in den

letzten 30 Tagen

$21 \%$

Wahnhafte Beschwerden

Halluzinationen insgesamt

Halluzinationen in den letzten 30 Tagen

Gedächtnisstörungen

Schwierigkeiten mit Verständnis, Gedächtnis und

Konzentration ingesamt

Schwierigkeiten mit Verständnis, Gedächtnis und

Konzentration in den letzten 30 Tagen

gewalttätiges Verhalten

Schwierigkeiten, gewalttätiges Verhalten zu

kontrollieren ingesamt

Schwierigkeiten, gewalttätiges Verhalten zu

kontrollieren in den letzten 30 Tagen

Suizid

Suizidgedanken insgesamt $51 \%$

Suizidgedanken in den letzten 30 Tagen $\quad 11 \%$

Suizidversuche insgesamt $35 \%$

Suizidversuche in den letzten 30 Tagen $\quad 2 \%$

Bei der Untersuchung der psychischen Gesundheit fiel bei $41 \%$ der Patienten ein akut schlechter bis sehr schlechter psychischer Zustand auf. Jeder fünfte Patient erhielt bei Eintritt regelmäßig Medikamente wegen einer psychischen Erkrankung (Tab.2).

In der Eintrittsbefragung gab die Hälfte der Patienten an, schon unter schweren Depressionen gelitten zu haben (Tab.3). Aktuell litten gemäß Eigenangaben 19\% der Patienten unter schweren Depressionen. Ein vergleichbares Bild fand sich bei den Fragen nach Angst und Spannungszuständen. Unter wahnhaften Beschwerden haben schon nach eigenen Angaben 8\% der Eintretenden gelitten. Über ein Drittel der Eintretenden hatte schon einmal einen Suizidversuch durchgeführt. Über die Hälfte gab an, schon an einen solchen Versuch konkret gedacht zu haben. 
Tab. 4 Prävalenz psychischer Erkrankungen nach ICD-10 in der heroingestützten Behandlung ( $n=77$; aus zwei Behandlungsstellen, Mehrfachdiagnose möglich)

\begin{tabular}{ll}
\hline F2 Schizophrenie & $9 \%$ \\
F3 affektive Störungen & $39 \%$ \\
F4 neurotische Störungen & $5 \%$ \\
F6 Persönlichkeitsstörungen & $68 \%$ \\
keine weitere psychische Störung & $3 \%$ \\
\hline
\end{tabular}

Tab. 4 gibt die Prävalenz von psychischen Erkrankungen nach ICD-10 in der heroingestützten Behandlung wieder. Untersucht wurden alle 77 Patienten zweier Behandlungsstellen durch zwei Fachärzte für Psychiatrie. Entsprechend den gehäuften, allgemeinen psychischen Störungen in der Eintrittsuntersuchung der Gesamtkohorte fand sich eine deutlich erhöhte Prävalenz von psychischen Erkrankungen. Bei 68\% der Patienten wurde eine Persönlichkeitsstörung, bei 39\% eine affektive Störung und bei 9\% eine Schizophrenie diagnostiziert. Nur bei zwei von 77 Patienten (3\%) wurde neben der Opiatabhängigkeit keine weitere psychische Störung gefunden.

\section{Psychische Entwicklung im Behandlungsverlauf}

Alle Patienten in der heroingestützten Behandlung wurden regelmäßig alle sechs Monate medizinisch untersucht. Im Behandlungsverlauf verbesserte sich bei rund der Hälfte der Patienten, welche in akut schlechtem Zustand eintraten, ihr psychischer Allgemeinzustand deutlich (Tab.5). Der aktuelle Behandlungsbedarf nach ärztlicher Einschätzung nahm aber nicht dementsprechend ab.

Parallel zu der Verbesserung des psychischen Allgemeinzustandes fand sich auch eine Abnahme der angegebenen psychischen Beschwerden gemäß Symptom-Check-List (SCL-90). Die durchgeführte Verlaufsanalyse über 18 Behandlungsmonate zeigt einen Rückgang der drei Syndrome Depression,
Tab. 5 Psychische Gesundheit im Behandlungsverlauf $(n=237)$

\begin{tabular}{lll}
\hline & bei Eintritt & $\begin{array}{l}\text { nach 18. Behandlungs- } \\
\text { monaten }\end{array}$ \\
\hline $\begin{array}{l}\text { psychischer Zustand } \\
\text { gut }\end{array}$ & $63 \%$ & $82 \%$ \\
$\begin{array}{l}\text { schlecht } \\
\text { md }=16 ; p \leq 0,001\end{array}$ & $36 \%$ & $18 \%$ \\
$\begin{array}{l}\text { Behandlungsbedarf } \\
\text { kein bis leichter Bedarf }\end{array}$ & $32 \%$ & \\
$\begin{array}{l}\text { mittlerer Bedarf } \\
\text { großer Bedarf }\end{array}$ & $47 \%$ & $38 \%$ \\
md=22; n.s. & $21 \%$ & $46 \%$ \\
& & $16 \%$ \\
\hline
\end{tabular}

Angst und Wahn sowie aggressives Agieren (Tab. 6). In der geschlechtsspezifischen Analyse der Beschwerdeangaben fanden sich Unterschiede. Männer gaben insgesamt weniger Beschwerden an, wobei die Abnahme der Beschwerdeangaben für Patientinnen wie Patienten denselben Verlaufstrend aufweist.

Um zu überprüfen, ob diese günstige Entwicklung der psychischen Gesundheit auch eine Veränderung der Hospitalisationshäufigkeit wegen psychischer Erkrankungen nach sich zieht, wurden retrospektiv von 651 Patienten, die Ende 1997 noch in der heroingestützten Behandlung verweilten, die Hospitalisationsdaten systematisch gesammelt. Erfasst wurden alle Klinikeinweisungen wegen psychischer Erkrankungen zwei Jahre vor und zwei Jahre während der heroingestützten Behandlung. Im Vergleich mit der Zeit vor der Behandlung zeigte sich, dass die Anzahl Spitalaufenthalte auf weniger als die Hälfte zurückging. Die Anzahl Spitalwochen ging auf weniger als ein Drittel des Basiswertes vor Behandlungsbeginn zurück (Tab. 7).

Bei der Analyse der zur Hospitalisation führenden Hauptdiagnosen fanden sich keine wesentlichen Veränderungen. Vor Eintritt in die heroingestützte Behandlung betrafen $85 \%$ der

\begin{tabular}{llll}
\hline & Eintritt & 12 Monate & 18 Monate \\
\hline $\begin{array}{l}\text { Beschwerdekategorien } \\
\text { depressive Beschwerden }\end{array}$ & & \\
hoch & $32 \%$ & $23 \%$ & $19 \%$ \\
mittel & $34 \%$ & $25 \%$ & $27 \%$ \\
tief & $34 \%$ & $52 \%$ & $54 \%$ \\
$12 / 18$ Mt. p=n.s. & & \\
Angst und wahnhafte Beschwerden & & & \\
hoch & $33 \%$ & $23 \%$ & $17 \%$ \\
mittel & $35 \%$ & $30 \%$ & $29 \%$ \\
tief & $33 \%$ & $47 \%$ & $54 \%$ \\
$12 / 18$ Mt. $\leq \leq 0,01$ & & & \\
aggressives Agieren & & & \\
hoch & $31 \%$ & $22 \%$ & $30 \%$ \\
mittel & $33 \%$ & $31 \%$ & $55 \%$ \\
tief & $36 \%$ & $48 \%$ & \\
$12 / 18$ Mt. $\mathrm{p} \leq 0,05$ & & &
\end{tabular}

Tab. 6 Angaben zu psychischen Beschwerden (SCL-90) in der heroingestützten Behandlung über 18 Monate $(n=233$; $m d=4$ a)

a $\mathrm{md}=$ missing data 


\begin{tabular}{llcc}
\hline & $\begin{array}{l}\text { in den 24 Monaten vor } \\
\text { Behandlungsbeginn }\end{array}$ & $\begin{array}{l}\text { in den 24 Monaten } \\
\text { während der Behandlung }\end{array}$ & $\mathrm{p}$-Wert \\
\hline Anzahl Spitalaufenthalte & 208 & 85 & $\mathrm{p} \leq 0,001$ \\
Anzahl Spitalwochen & 630 & 190 & $\mathrm{p} \leq 0,001$ \\
\hline
\end{tabular}

Tab. 7 Anzahl Hospitalisationen wegen psychischer Erkrankungen bei 651 Patienten über 4 Jahre
Spitaleintritte die Hauptdiagnose „Psychische Störung durch psychotrope Substanz“ (F10 bis F19). In der heroingestützten Behandlung waren es $88 \%$. Da jeweils nur die unmittelbar zur Hospitalisation führende Hauptdiagnose erfasst wurde, kann hier keine weitergehende Aussage zu zusätzlichen psychischen Störungen gemacht werden.

Insgesamt zeigen die durchgeführten Analysen eine erhebliche Verbesserung der psychischen Gesamtsituation der Patienten in der heroingestützten Behandlung. Die Verbesserung konnte schon in den ersten Monaten festgestellt werden und setzt sich zumindest teilweise auch nach einem Behandlungsjahr weiter fort.

\section{Diskussion}

Im Rahmen der schweizerischen Studien zur heroingestützten Behandlung war es möglich, in den Jahren 1994 bis 1996 eine Kohorte von über 1000 Opiatabhängigen bei Eintritt und im Verlauf zu untersuchen. Die hier vorliegende Arbeit beschreibt die psychische Gesundheit aller Eintretenden und den Behandlungsverlauf bei einer repräsentativen Stichprobe.

Bei der Interpretation der vorliegenden Resultate muss berücksichtigt werden, dass die Patienten in der heroingestützten Behandlung nicht repräsentativ für alle Opiatkonsumenten sind. In die Studie wurden gezielt Opiatabhängige aufgenommen, die bisher nicht erfolgreich behandelt werden konnten und erhebliche sozial-medizinische Defizite aufwiesen. Dementsprechend wird eine selektive Gruppe von langjährigen Opiatabhängigen mit schweren sozialen und medizinischen Problemen beschrieben, welche mit der beschriebenen ambulanten heroingestützten Behandlung erreicht werden konnte.

Bei der Diskussion der Verlaufsresultate muss weiter berücksichtigt werden, dass keine im gleichen Umfang untersuchte Kontrollgruppe zum Vergleich vorliegt. Für Teilkohorten konnten Vergleichsgruppen gebildet werden [21]. Es zeigte sich eine bessere Wirkung des intravenös applizierten Heroins im Vergleich mit Morphin i.v. und Methadon i.v. [8].

Trotz der genannten Einschränkungen ergeben die vorhandenen Daten wichtige Informationen über die Situation langjähriger Opiatabhängiger vor und in der heroingestützten Behandlung und über die Effekte dieser neuen Therapie auf die psychische Gesundheit.

Die Analyse der Eintrittsdaten zeigt, dass eine Gruppe von langjährigen Opiatabhängigen mit multiplen sozialen und medizinischen Problemen erreicht werden konnte. Über $40 \%$ der Patienten waren bei Eintritt in akut schlechtem oder sehr schlechtem psychischen Zustand gemäß ärztlicher Beurteilung. Analog dazu fanden sich in der Eintrittsanamnese verschiedenste Hinweise auf aktuelle psychische Probleme. So gab rund jeder fünfte Patient bei Eintritt an, in den letzten Wochen unter einer schweren Depression gelitten zu haben. Jeder dritte Patient hat gemäß den Eintrittsangaben schon einen Suizidversuch unternommen.

Entsprechend zeigen sich gehäuft psychische Störungen gemäß ICD-10. Besonders auffällig, aber aufgrund der bekannten Literatur gut nachvollziehbar, ist der hohe Anteil von Persönlichkeitsstörungen und affektiven Störungen. In der Literatur finden sich bei Opiatabhängigen vergleichbare Prävalenzen von 50 bis $90 \%$ bei Persönlichkeitsstörungen, 20 bis $60 \%$ bei affektiven Störungen und 0 bis $20 \%$ bei psychotischen Störungen. Auffällig ist weiter, dass $97 \%$ der behandelten Patienten neben der Substanzabhängigkeit eine weitere psychische Störung aufwiesen. Die hier beschriebene Prävalenz von psychischen Erkrankungen ist charakteristisch für die Situation bei Patienten in der heroingestützten Behandlung der Schweiz. So fanden sich in einer 1997 durchführten Untersuchung bei den 186 Patienten eines großstädtischen Behandlungszentrums vergleichbare Erkrankungshäufigkeiten [22]. Einzig die Prävalenz von Persönlichkeitsstörungen lag mit über $85 \%$ noch höher.

Im Behandlungsverlauf über 18 Monate zeigt sich bei den in der Therapie verbleibenden Patienten eine deutliche Verbesserung des psychischen Zustandes gemäß ärztlicher Beurteilung, aber auch gemäß der subjektiver Symptomangabe. Im Behandlungsverlauf werden deutlich weniger depressive, ängstliche und wahnhafte Beschwerden angegeben. Auch das aggressive Verhalten geht gemäß Selbstangaben zurück. Die Besserung tritt schon im ersten Behandlungsjahr auf und verstärkt sich noch leicht im zweiten Behandlungsjahr.

Um die gemäß ärztlicher Beurteilung und Patientenangaben gefundene günstige Entwicklung weiter beschreiben zu können, wurde 1997/98 eine retrospektive Erfassung der Hospitalisationsdaten zwei Jahre vor und in den ersten zwei Jahren in der heroingestützten Behandlung durchgeführt. Entsprechend zu den gefundenen günstigen Effekten in der heroingestützten Behandlung fand sich ein Rückgang der Hospitalisationen bei den in der Behandlung verbliebenen Patienten. Die Anzahl Spitalaufenthalte ging auf weniger als die Hälfte, die Anzahl Spitalwochen auf weniger als ein Drittel der Situation vor Behandlungsbeginn zurück. In einer zusätzlichen gesundheitsökonomischen Studie konnte gezeigt werden, dass im Behandlungsverlauf der Rückgang von affektiven Störungen auch mit einem Einsparungseffekt bei den ambulanten und stationären Kosten einhergeht $[23,24]$. Dies sollte besonders berücksichtigt werden, wenn die Kosteneffizienz dieser neuen Behandlungsform diskutiert wird.

Insgesamt konnte in den bisherigen Analysen der heroingestützten Behandlung eine erhebliche Verbesserung der psychischen Gesundheit der Patienten nachgewiesen werden. Die heute vorliegenden Ergebnisse zeigen aber auch, dass mit 
der heroingestützten Behandlung eine Patientengruppe mit schweren psychischen Störungen erreicht wird. Dies stellt zweifellos hohe Anforderungen an die durchgeführten Behandlungen, da gerade im ambulanten Behandlungssetting die Diagnostik und Therapie bei Drogenabhängigen mit zusätzlichen psychischen Störungen einen erheblichen fachlichen und zeitlichen Aufwand erfordert.

Die bisherigen Erfahrungen in der heroingestützten Behandlung zeigen, dass der Optimierung der psychiatrischen Behandlung besondere Aufmerksamkeit geschenkt werden sollte. Deshalb soll die psychiatrische Diagnostik und Therapie in einem behandlungsstellen-übergreifenden Prozess zukünftig weiter optimiert werden. Angestrebt wird in einem ersten Schritt eine standardisierte, psychiatrischen Diagnostik mit einheitlichen Instrumenten in allen Behandlungsstellen. Basierend auf diesen laufend erhobenen, standardisierten Daten soll dann ein eigentlicher Behandlungs- und Forschungsverbund aufgebaut werden. So kann eine breite Grundlage für die Weiterentwicklung von auf EvidenceBased-Medicine-Kriterien basierenden Therapien in der psychiatrischen, ambulanten Behandlung von Drogenabhängigen gelegt werden.

Dadurch sollte es möglich sein, die gefundenen, günstigen Effekte der heroingestützten Behandlung auf die Gesundheit der Teilnehmenden weiter zu verstärken. Mit dem anlaufenden Prozess ist aber auch die Hoffnung verbunden, dass damit auch ein weiterer Beitrag zur allgemeinen Verbesserung der Therapie von psychischen Störungen bei Drogenabhängigen geleistet werden kann.

\section{Literatur}

${ }^{1}$ Gölz J. Der drogenabhängige Patient. München: Urban \& Fischer, 1999

2 Krausz M, Verthein U, Degwitz P. Komorbidität - Psychische Störungen und Symptome bei Opiatabhängigen. In: Backmund M, Soyka M (Hrsg). 1. Interdisziplinärer Kongress für Suchtmedizin, Landsberg: verlag moderne industrie AG, 1999: 166-173

${ }^{3}$ Christie KA, Burke JD, Regier DAet al et al. Epidemiologic evidence for early onset of mental disorders and higher risk of drug abuse in young adults. Am J Psychiatry. 1988; 145: 971-975

4 Regier DA, Farmer ME, Rae DSet al et al. Comorbidity of mental disorders with alcohol and other drug abuse. Results from the Epidemiologic Catchment Area (ECA) Study. Jama 1990; 264: 2511-2518

${ }^{5}$ Wittchen HU, Perkonigg A, Reed V. Comorbidity of Mental Disorders and Substance Use Disorders. Eur Addict Res 1996; 2: 3647

${ }^{6}$ Mino A. Analyse scientifique de la littérature sur la remise contrôlée d'héroïne ou de morphine. Bern: Bundesamt für Gesundheit, 1990

7 Steffen T, Kaufmann B, Blättler R et al. Die heroingestützte Behandlung von Opiatabhängigen - bisherige und aktuelle Forschungsschwerpunkte. Das Gesundheitswesen 1999; 61: 407412

8 Uchtenhagen A, Dobler-Mikola A, Steffen T et al. Prescription of Narcotics for Heroin Addicts - Main Results of the Swiss National Cohort Study. Basel: Karger, 1999

9 Uchtenhagen A, Gutzwiller F, Dobler-Mikola A (Hrsg). Versuche für eine ärztliche Verschreibung von Betäubungsmitteln - Synthesebericht. Zürich: ISF/ISPMZ-Bericht, 1997
${ }^{10}$ Drucker E, Vlahov E. Controlled clinical evaluation of diacetyl morphine for treatment of intractable opiate dependence. Lancet. 1999; 353: 1543-1544

${ }^{11}$ Farell M, Ward W. The Swiss Heroin trials: testing alternative approaches (Editorial). Brit Med J. 1998; 316: 639

12 Krausz M, Behrendt K. Ärztlich kontrollierte Heroinverschreibung als Teil differenzierter Suchttherapie. Sucht 1998; 44: 218-219

13 Soyka M. Zum Problem der ärztlich kontrollierten Heroinvergabe an Drogenabhängige. Sucht 1998; 44: 220-222

${ }^{14}$ WHO. External Evaluation Panel: Report of the External Panel on the Evaluation of the Swiss Scientific Studies of Medically Prescribed Narcotics to Drug Addicts. Geneva: Substance Abuse Department WHO, 1999

${ }^{15}$ Blanken P, Henriks V, Pozzi G et al. European Addiction Severity Index EuropASI. A guide to training and administering EuropASI interviews. COST A6 Brussels, 1994

${ }^{16}$ WHO. International Statistical Classification of Diseases and Related Health Problems. WHO Geneva: Tenth Revision, 1992

17 Derogatis LR, Cleary PA. Confirmation of the dimensional structure of the SCL-90: a study in construct validation. J Clin Psychol. 1977; 33: 981-989

18 Rihs-Middel M. Heroinverschreibung im Spannungsfeld von Forschung und Praxis. In: Westermann B, Bellmann GU, Jellinek C (Hrsg). Heroinverschreibung - Wirkungen und Nebenwirkungen, Weinheim: Deutscher Studienverlag/Beltz, 1999: 191-199

${ }^{19}$ Kaufmann B, Steffen T, Dobler-Mikola A et al. Die Praxis der heroingestützten Behandlung - erste Ergebnisse der Behandlungsforschung. In: Westermann B. Bellmann GU, Jellinek C (Hrsg). Heroinverschreibung - Wirkungen und Nebenwirkungen, Weinheim: Deutscher Studienverlag/Beltz, 1999: 191-199

20 SPSS Inc. SPSS Base System User's Guide. Chicago: SPSS Inc, 1994

21 Perneger T, Giner F, del Rio M, Mino A. Heroin maintenance programme for addicts who fail in conventional drug treatments: a randomised trial. BMJ 1998; 317: 13-18

22 Stohler R, Ladewig D. Sind Doppeldiagnosen-Abteilungen geeignete Einrichtungen zur Behandlung von Patient(inn)en mit Störungen durch psychotrope Substanzen und zusätzlichen psychiattrischen Störungen? Abhängigkeiten. 1997; 3: 19-24

${ }^{23}$ Frei A, Steffen T, Gasser M et al. Gesundheitsoekonomische Bewertung der Versuche für eine ärztliche Verschreibung von Betäubungsmitteln (PROVE). Soz Präventivmed. 1998; 43: 185194

${ }^{24}$ Gutzwiller F, Steffen T (Hrsg). Cost Benefit Analysis of Heroin Maintenance Treatment. Basel: Karger, 2000

\section{Dr. Thomas Steffen, MPH}

Forschungsleiter am Institut für Suchtforschung Konradstraße 32

8005 Zürich

Schweiz

E-mail: steffen@isf.unizh.ch 